\title{
Phonological factors in STM: Similarity and the unattended speech effect
}

\author{
PIERRE SALAMÉ \\ CNRS/INRS Laboratoire de Physiologie et de Psychologie Environnementales, Strasbourg, France
}

and

\author{
ALAN BADDELEY \\ MRC Applied Psychology Unit, Cambridge, England
}

\begin{abstract}
Immediate memory for sequences of 5, 6, 7, or 8 phonologically similar or dissimilar consonants was studied using visual presentation accompanied by silence or continuous speech in an unfamiliar language, Arabic. There were significant effects of list length, phonological similarity, and unattended speech, and significant interactions between similarity and unattended speech and between similarity and list length. The interactions are shown to stem primarily from the absence of a decrement due to phonological similarity at List Length 8 . It is suggested that this absence is attributable to a strategy of abandoning phonological coding when performance drops below some minimum level.
\end{abstract}

There is abundant evidence that the immediate serial recall of verbal materials tends to rely heavily on phonological coding, an association that is explained within the working memory framework in terms of a subsidiary slave system, the articulatory loop (Baddeley, in press; Salamé \& Baddeley, 1982). This model assumes a phonological store and an articulatory control process. Speech-based information can be registered in the store either by an optional process of subvocalization or by an obligatory registration through hearing spoken material.

The model accounts for a range of phenomena, including the phonological similarity effect and the unattended speech effect. Sequences of phonologically similar items are harder to recall than dissimilar sequences (Conrad \& Hull, 1964); this is assumed to be because the constituent items have similar, and hence confusable, codes within the store. Unattended speech disrupts the retention of sequences of visually presented items (Colle \& Welsh, 1976; Salamé \& Baddeley, 1982); this is assumed to be because the unattended spoken material gains obligatory access to the phonological store, hence corrupting the memory trace left by the visually presented items that are entered into the store through subvocal rehearsal. In line with this interpretation, using articulatory suppression to prevent subvocal rehearsal impairs performance and also removes the unattended speech effect (Salamé \& Baddeley, 1982). The way in which the memory trace is corrupted by unattended speech is, however, unspecified. The experiment described here attempts to explore this issue by combining the effects of phonological similarity with those of unattended speech.

We are grateful to Ian Nimmo-Smith for statistical advice. Address correspondence to: P. Salamé, CNRS/INRS, Laboratoire de Physiologie et de Psychologie Environnementales, 21 Rue Becquerel, F-67087 Strasbourg Cedex, France.
What might one predict as a result of combining these two variables? Unfortunately we do not have a sufficiently well worked out model of the operation of the phonological store to make firm predictions. One possibility is that the memory traces of the similar items, having fewer distinguishing features, might be more susceptible to disruption by unattended speech. On the other hand, an interaction in the opposite direction might occur if one assumes that both unattended speech and phonological similarity are causing an impairment of the same limited component of performance. If, for example, the phonological similarity effect is great enough to remove the contribution to performance of the phonological store, then further disruption of the phonological store by unattended speech would be irrelevant. The abolition of the unattended speech effect by articulatory suppression can be explained in this way (Salamé \& Baddeley, 1982).

Colle and Welsh (1976) studied the influence of continuous unattended speech on immediate memory for sequences of eight consonants that were either phonologically similar or dissimilar. They observed an interaction, with an unattended speech effect occurring only for the dissimilar items. The simplest interpretation of this result is to assume that both unattended speech and phonological similarity tend to wipe out the phonological contribution to performance. Once the component is obliterated, further disruption of the phonological component is irrelevant.

In an initial attempt to replicate this result, we observed no interaction between phonological similarity and unattended speech when subjects recalled sequences of six similar or dissimilar consonants. We therefore decided to explore the matter further by carrying out a study in which sequence length was increased systematically from five to eight similar or dissimilar consonants, visually 
presented either in silence or with continuous unattended speech.

\section{METHOD}

The material comprised sequences of $5,6,7$, or 8 consonants selected at random from one of two sets, dissimilar (F H K L N Q X Z) or similar (B C D G P T V W; in French, W rhymes with the preceding letters, whereas $Z$ does not). The items were presented $2 \mathrm{sec}$ after a visual warning signal at a rate of $500 \mathrm{msec}$ per letter, followed by a blank interval of $250 \mathrm{msec}$. The letters were $1 \mathrm{~cm}$ in height, were uppercase, and appeared in green at the center of a black screen. All subjects were tested in ascending order of sequence length in a blocked design in which each block comprised 12 similar and 12 dissimilar lists in alternating order. Before each block, the subject was given a 5-sec cue indicating the length of sequence to follow. Subjects were seated $1 \mathrm{~m}$ from the screen, and were instructed to write down their responses immediately after the end of the sequence, in the order in which they had been presented in an answer booklet. They were allowed $13 \mathrm{sec}$ per sequence, and were asked to remain silent throughout the test.

All subjects were initially given a 10-min pretest in quiet, and on the basis of their performance, subjects were assigned to one of two matched groups. One group was tested first in quiet and then in speech; the other group was tested in the reverse order. The speech condition involved a continuous reading from an Arabic work (The Prophet by G. K. Gibran); it was presented through two Lansing loudspeakers at a mean level of $75 \mathrm{~dB}(\mathrm{~A})$. The ambient sound level for the quiet control condition was $37 \mathrm{~dB}(\mathrm{~A})$. A total of 20 introductory psychology students from the University of Strasbourg, all of whom were unfamiliar with Arabic, took part in the experiment and were paid for participation.

\section{RESULTS}

Figure 1 shows the mean number of items recalled in the correct serial position as a function of sequence length, phonological similarity, and unattended speech. There was no effect of order of test $[F(1,16)<1]$ and no interaction of order with any other variable. There were significant effects of phonological similarity $[F(1,16)=17.33$, $p<.001]$, of unattended speech $[F(1,16)=27.10$, $p<.001]$, and of sequence length $[F(3,48)=174.69$, $p<.001]$. Significant interactions occurred between phonological similarity and unattended speech $[F(1,16)$ $=5.16, p<.05]$ and between phonological similarity and list length $[F(3,54)=4.67, p<.01]$.

Because of the interaction with list length, we performed further separate analyses at each length. For List Length 5 , there were significant effects of phonological

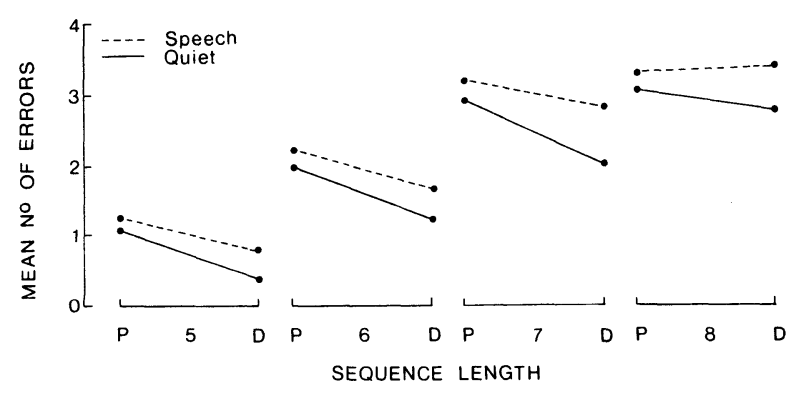

Figure 1. Serial recall of phonologically similar $(P)$ and dissimilar (D) consonant sequences presented visually in quiet or unattended speech as a function of sequence length. similarity $[F(1,18)=14.24, p<.01]$ and of unattended speech $[F(1,18)=5.66, p<.05]$, but no significant interaction between these two variables $[F(1,18)=1.58$, $p>$.05]. For List Length 6, the pattern of results was broadly similar, with a highly significant effect of phonological similarity $[F(1,18)=15.90, p<.01]$ and a significant effect of unattended speech $[F(1,18)=6.15$, $p<.05$ ], but again with no trace of an interaction $[F(1,18)<1]$. At Length 7 there were again significant effects of phonological similarity $[F(1,18)=12.72$, $p<.01]$ and unattended speech $[F(1,18)=19.67$, $p<.01]$. There was some suggestion of an interaction between similarity and unattended speech, although this did not reach statistical significance $[F(1,18)=3.04$, $p>.05]$. For sequences of 8 items, the pattern was clearly different, with the effect of phonological similarity failing to reach significance $[F(1,18)<1]$, and the unattended speech effect remaining strong $[F(1,18)=$ $19.30, p<.01]$. Again, there was a suggestion of an interaction between these two variables, but this did not reach significance $[F(1,18)=2.39, p>.05]$. All of these analyses show significant effects of serial position, but only one of the eight interactions between major variables and serial position reached significance, that between phonological similarity and serial position for List Length $5[F(4,72)=5.30, p<.01]$.

\section{DISCUSSION}

Our results suggest that although both unattended speech and phonological similarity influence immediate memory for visually presented consonants, they do so in somewhat different ways. The unattended speech effect is present for all lengths, being relatively weak for short sequences, but remaining clear up to sequences of Length 8 . On the other hand, phonological similarity has a clear effect on short sequences, but becomes somewhat less clear-cut at Length 7 and is no longer statistically significant at Length 8 .

The disappearance of the effect of phonological similarity at List Length 8 has two interesting parallels with earlier literature. First, Colle and Welsh (1976) report an interaction between the effects of unattended speech and phonological similarity based on a study using 8-item sequences. They observed an effect of phonological similarity in quiet, but not when unattended speech was presented.

A second parallel is with research on the susceptibility of poor readers to the effects of phonological similarity. A number of studies have indicated that children who show delayed acquisition of reading tend to have impaired memory span and to be less sensitive to the effects of phonological similarity than do good readers (I. Y. Liberman, Shankweiler, A. M. Liberman, Fowler, \& Fischer, 1977). This phenomenon has not always proved easy to replicate (see Baddeley, 1986, for a review), and Hall, Wilson, Humphreys, Tinzmann, and Bowyer (1983) have suggested that the lack of susceptibility to the effects of phonological similarity is characteristic of subjects performing at the limit of their memory spans. Typically, good and poor readers are presented with letter sequences of the same length; since poor readers normally have impaired span, they are more likely to be operating nearer to the limit of performance, and this may be why they are more likely to show an absence of the phonological similarity effect.

How, then, should our own results be interpreted? For sequences up to and including seven items, phonological similarity and unattended speech appear to have broadly additive effects. A superficial application of the additive factors logic devised by Sternberg (1969) might suggest that our results imply that these two components affect different components of the memory system. Note, however, that the Sternberg analysis is valid only under highly constrained conditions, which as- 
sume that the measure used is reaction time rather than errors and that the system is one in which a series of successive stages occur, with each stage being required to complete its processing before passing on the necessary signal to the following stage. These assumptions are of course not valid in the case of immediate memory paradigms, such as that used here, where stages are almost certainly not independent, and where error rather than reaction time measures are taken. As Broadbent (1984) has pointed out, the presence or absence of an interaction is only interpretable within the context of a clearly specified model. No such model currently exists in the case of the analysis of the articulatory loop; however, our observation of additive effects of phonological similarity and unattended speech place clear constraints on any such model that is developed.

How should we explain the pattern of results for sequences of eight letters? The most obvious interpretation here would seem to be via a change in strategy. Provided the phonological coding strategy is capable of supporting performance at a reasonably high level, then it is sensible for the subject to keep to it. When performance drops below some minimum, such as $50 \%$, as it does for sequences of eight similar consonants, then it is not unreasonable to abandon such a strategy, possibly in favor of either a visual or a semantic strategy. When the eight consonants are dissimilar, subjects are still performing at a level of more than $50 \%$ correct, and hence might be assumed to continue using a phonological coding strategy. This assumption would account for the continued

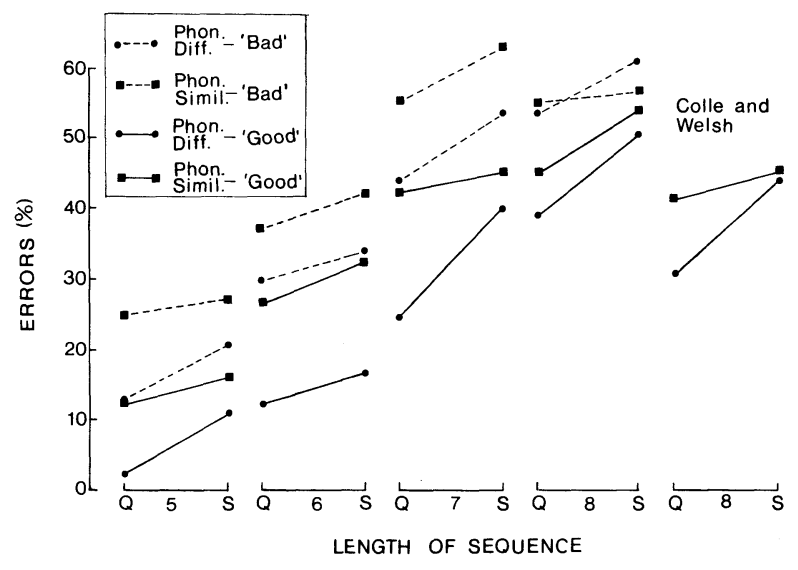

Figure 2. The effects of phonological similarity and unattended speech on the recall of consonant sequences by subjects who were above the average ("good") or below the average ("bad") in pretest memory performance. Data from a similar study by Colle and Welsh (1976) are included for comparison. occurrence of the unattended speech effect with sequences of eight dissimilar consonants. Some limited support for this view is given in Figure 2, which shows the performance of our subjects separated into those whose scores are above the mean, and those whose scores are below it. "Good" subjects show a pattern of performance at List Length 8 that resembles performance on shorter lists, while "bad" subjects show evidence of abandoning phonological coding at Length 8 .

The tendency for "bad" subjects to remember slightly more items in the condition in which the effects of similarity are added to those of unattended speech suggests that this may be an effective strategy. Clearly, such an interpretation is somewhat speculative since we have no direct evidence of the alternative form of coding that might be in use; this certainly would appear to merit further investigation, particularly in view of its possible role in the controversy surrounding the role of phonological coding in poor readers.

In conclusion, our results indicate that the effects on immediate serial recall of unattended speech and phonological similarity are additive for sequences of five to seven consonants. At Length 8, the effect of similarity is attenuated, particularly under concurrent unattended speech (Colle $\&$ Welsh, 1976) and with subjects of below average memory performance.

\section{REFERENCES}

Baddeley, A. D. (1986). Working memory. Oxford University Press. Broadbent, D. E. (1984). The Maltese Cross: A new simplistic model for memory. Behavioral \& Brain Sciences, 7, 55-94.

Colle, H. A., \& Welsh, A. (1976). Acoustic masking in primary memory. Journal of Verbal Learning \& Verbal Behavior, 15, 17-32.

ConRad, R., \& Hull, A. J. (1964). Information, acoustic confusion and memory span. British Journal of Psychology, 55, 429-432.

Hall, J. W., Wilson, K. P., Humphreys, M. S., Tinzmann, M. B., \& BOWYER, P. M. (1983). Phonemic-similarity effects in good vs. poor readers. Memory \& Cognition, 11, 520-527.

Liberman, I. Y., Shankweiler, D., Liberman, A. M., Fowler, C., \& FisCher, F. W. (1977). Phonetic segmentation and recoding in the beginning reader. In A. S. Reber \& D. Scarborough (Eds.), Toward a psychology of reading (pp. 207-226). Hillsdale, NJ: Erlbaum.

Salamé, P., \& Baddeley, A. D. (1982). Disruption of short-term memory by unattended speech: Implications for the structure of working memory. Journal of Verbal Learning \& Verbal Behavior, 21, 150-164.

STERNBERG, S. (1969). The discovery of processing stages: Extensions of Donders' Method. Acta Psychologica, 30, 276-315.

(Manuscript received for publication March 18, 1986.) 